\title{
Gamificação como ferramenta para melhoria do ensino e aprendizagem: uma análise bibliométrica
}

\section{Gamification as a tool for improving teaching and learning: a bibliometric analysis}

\author{
1 Cleide Ane Barbosa da Cruz cleianebar@gmail.com \\ 2 Vinicius Marques Nejaim \\ 3 Ana Katia Lima da Costa \\ 3 Leonardo Santos Sousa \\ ${ }^{3}$ Washington Martins da Costa Junior
}

1 Doutora em Ciência da Propriedade Intelectual pela UFS (2020). Atualmente é professora substituta do Curso Técnico de Comércio do IFS Campus Tobias Barreto. Instituto Federal de Sergipe.

2 Doutorando em Ciência da Propriedade Intelectual/ Universidade Federal de Sergipe. Professor do Instituto Federal de Sergipe Campus Itabaiana. Instituto Federal de Sergipe.

3 Graduanda(o) em Tecnologia em Logística - Instituto Federal de Sergipe.

\section{Resumo}

A gamificação corresponde à utilização de elementos de jogos que proporcionam divertimento e influenciam no comportamento do jogador em um contexto de não jogo. Por isso, esta pesquisa tem como objetivo apresentar uma análise bibliométrica de publicações científicas sobre gamificação, com o intuito de destacar sugestões para aplicação no ensino e aprendizagem dos alunos. Com relação à metodologia, a pesquisa se classifica como exploratória quantitativa. Foi realizada uma análise bibliométrica por meio de busca nas bases Scielo e Scopus utilizando-se a palavra-chave gamificação e gamification, sendo encontradas 15 publicações na base Scielo e 564 publicações na base Scopus, entre os anos de 2012 a 2019. Após análise dos artigos, pode-se observar que houve um aumento significativo de publicações referentes ao tema nos últimos anos. Percebeu-se que a área temática Ciências Sociais apresentou maior número de publicações, sendo que a de Negócios, Gestão e Contabilidade está entre as 10 áreas com mais produções voltadas ao tema, representando $41,71 \%$ dos artigos encontrados. Houve uma maior concentração de publicações dos Estados Unidos sobre o tema em estudo. Dessa forma, notou-se que a verificação da bibliometria possibilita que se consultem publicações sobre determinado tema, auxiliando pesquisadores em suas pesquisas futuras. Além disso, identificou-se, com esta pesquisa, que essa técnica permite que professores utilizem elementos de jogos com o propósito de impulsionar e motivar os alunos a uma melhor aprendizagem.

Palavras-chave:

Educação. Jogos. Professor.

\begin{abstract}
Gamification corresponds to the use of game elements that provide fun and influence the player's behavior in a non-game context. Therefore, this research aims to present a bibliometric analysis of scientific publications on gamification, in order to highlight suggestions for application in the teaching and learning of students. Regarding the methodology, the research is classified as quantitative exploratory. A bibliometric analysis was performed by searching the Scielo and Scopus databases using the keyword gamificação and gamification, with 15 publications in the Scielo database and 564 publications in the Scopus database, between the years 2012 to 2019. After analyzing the articles, it can be seen that there has been a significant increase in publications on the subject in recent years. It was noticed that the Social Sciences thematic area presented a greater number of publications, being that of Business, Management and Accounting is among the 10 areas with more productions focused on the theme, representing $41.71 \%$ of the articles found. There was a greater concentration of publications from the United States on the topic under study. Thus, it was noted that checking bibliometrics makes it possible to consult publications on a certain topic, assisting researchers in their future research. In addition, it was identified, with this research, that this technique allows teachers to use elements of games with the purpose of boosting and motivating students to better learning.
\end{abstract}

\section{Keywords:}

Education. Games. Teacher.

Como você deve citar?

CRUZ, Cleide Ane Barbosa da et al. Gamificação como ferramenta para melhoria do ensino e aprendizagem: uma análise bibliométrica. Cadernos UniFOA, Volta Redonda (RJ), v. 16, n. 46, p. 1-12, ago, 2021. 


\section{INTRODUÇÃO}

Com o passar dos anos a gamificação vem sendo pesquisada e aprimorada, englobando diversas áreas, como a educação e o cenário empresarial. Baseia-se no uso de algumas ferramentas específicas que auxiliam na resolução de problemas e melhoram o aprendizado, motivando ações e comportamentos em diversos ambientes (SALAMI et al., 2018).

Além disso, a gamificação nada mais é que a utilização de algumas ferramentas específicas que ajudam a solucionar problemas e a melhorar o aprendizado, motivando ações e comportamentos em cenários fora do contexto de jogos. Tem como principal objetivo despertar a curiosidade e aumentar o engajamento dos usuários e, além de propor desafios em forma de jogos, traz recompensas que são itens cruciais para se obter o sucesso (DETERDING et al., 2011).

Dessa forma, tem o objetivo de gerar ou permitir a adaptação da experiência do usuário a um serviço, processo ou produto, visando à liberação de emoções positivas, motivação, engajamento das pessoas e a possibilidade de exploração das habilidades (PANTOJA; PEREIRA, 2018).

Em relação à bibliometria, busca-se analisar as atividades científicas ou técnicas por meio de estudos quantitativos das publicações, sendo um método flexível para se avaliar a quantidade das fontes de informação (SILVA; HAYASHI, 2011).

Diante disso, justifica-se a pesquisa pela necessidade de compreender o crescimento das publicações científicas em relação à utilização da gamificação, bem como destacar sugestões que possam ser aplicadas para impulsionar o ensino e a aprendizagem dos alunos nas escolas.

Dessa forma, esta pesquisa tem como objetivo apresentar uma análise bibliométrica de publicações científicas sobre gamificação, com o intuito de destacar sugestões para aplicação no ensino e aprendizagem dos alunos.

\section{CONCEITOS DE GAMIFICAÇÃO}

Gamificação envolve a utilização de elementos dos games, como mecânicas, estratégias, pensamentos que estão fora do contexto dos jogos, visando impulsionar a motivação dos indivíduos à ação, para auxiliar na solução de problemas e na promoção de aprendizagens (FARDO, 2013).

Além disso, estimula o pensamento crítico como em um jogo propriamente dito, contudo os principais objetivos são resolver problemas reais, melhorar continuamente os processos e criar um ambiente com o intuito de envolver e engajar os estudantes.

É importante ressaltar que quando participamos de um processo gamificado não significa que estamos participando de um jogo necessariamente, mas sim que estamos adquirindo suas características mais eficientes para conseguir os mesmos benefícios alcançados pelos jogos (VIANNA, 2013).

Ainda, as atividades gamificadas podem engajar públicos diferentes e com idades diversas. E o engajamento está diretamente ligado à relevância dos conteúdos, às pessoas e a forma como a aprendizagem é motivada (ALVES, 2015) 
Dessa forma, a ideia de que a gamificação favorece o engajamento dos estudantes em atividades escolares tidas por eles como entediantes, o que é inevitável, pois o uso dos games pode aproximar o processo de aprendizagem do estudante a sua própria realidade (TOLOMEI, 2017).

\section{GAMIFICAÇÃO NA EDUCAÇÃO}

A educação vem passando por diferentes desafios, necessitando acompanhar a evolução da sociedade contemporâneas e buscando criar rotinas que possibilitem a criação de um ambiente de aprendizagem que desperte o desenvolvimento pessoal e intelectual do aluno. Por isso, a gamificação surge como uma alternativa para agregar diferentes modos, ou seja, multimodalidade, visando captar o interesse dos alunos por meio da reinvenção do aprendizado (ORLANDI et al., 2018).

Esse fenômeno vem sendo disseminado pela educação, sendo utilizado como estratégia de ensino e aprendizagem, direcionado a um público-alvo inserido na chamada geração gamer, tendo apresentado resultados positivos, por meio dessa experiência (SHELDON, 2012).

Esse conceito é recente e foi introduzido no início dos anos 2000, mas sua utilização apenas cresceu a partir de 2010. Desde então, vem sendo aplicado em diferentes áreas, como finanças, saúde, educação, entre outros (GROH, 2012). Além disso, a gamificação corresponde à utilização das características de um jogo, como, por exemplo, o divertimento, influenciando o comportamento do jogador em um contexto de não jogo, o ambiente de trabalho. Para isso, utiliza técnicas de jogos com o propósito de impulsionar a motivação das pessoas (WU, 2011).

Em complemento, entende-se que a aplicação desse conceito nos ambientes colaborativos pode ser um importante incentivo para a busca da inovação e da gestão do conhecimento, bem como a aprendizagem em grupo, possibilitando também sua aplicação na educação para o desenvolvimento da aprendizagem dos alunos (FORMANSKI; ALVES, 2015).

Dessa forma, a gamificação possibilita o desenvolvimento das práticas educacionais, permitindo a melhoria do ensino e aprendizagem dos alunos com inserção de elementos de jogos que impulsionam novas formas dos professores aplicarem atividades e passarem conteúdo aos alunos na escola.

\section{GAMIFICAÇÃO APLICADA À ESCOLA}

Atualmente, a aplicação da gamificação na educação formal permite que se encontrem indivíduos que já possuam aprendizagens vindas das interações com os games. Além disso, possibilita que, na educação, se encontrem novas estratégias que são necessárias para atuar com esses indivíduos que estão cada vez mais inseridos no contexto das mídias e das tecnologias digitais e vem se mostrando desinteressados pelos métodos passivos existentes no ensino utilizado nas escolas (FARDO, 2013).

Em relação aos ambientes de aprendizagem, existem os presenciais e os virtuais. A gamificação, como foi citado anteriormente, apresenta elementos de jogos para utilização nesses ambientes de aprendizagem, porém não significa a obrigatoriedade da utilização de tecnologias para a obtenção dos objetivos no processo educativo. Ademais, a utilização do conceito de multimodalidade possibilita que o ambiente, seja virtual ou presencial, proporcione a imersão dos indivíduos na busca pela aprendizagem (KAPP, 2012).

Em contrapartida, existem iniciativas mais robustas que demandam a elaboração de plataformas virtuais, contratação de profissionais especializados que ajudem na elaboração do processo, no qual 
os alunos e docentes têm a possibilidade de levar a termo o processo de aprendizagem de forma mais significativa (ORLANDI; DUQUE; MORI, 2018).

Além disso, no contexto da sua aplicação na escola, a gamificação possibilita o desenvolvimento de atividades divertidas que podem atrair diferentes públicos com idades diversas. É importante ressaltar que a participação está ligada à relevância dos conteúdos que são passados às pessoas ou, no caso das escolas, aos alunos, e também pela forma como a aprendizagem é motivada pelos professores (ALVES, 2015).

Em relação às possibilidades de interação entre alunos e professores, entende-se que existem inúmeras formas de incentivar essa interação, como, por exemplo, estimular a participação dos alunos na criação de jogos que impulsionem a aprendizagem de um determinado conteúdo ou até mesmo a produção de artigos que, posteriormente, podem ser publicados em formato de livro. Por isso, a gamificação surgiu como meio de fortalecer o ensino e a aprendizagem e estimular a participação dos alunos no ambiente escolar (INHELORA, 2002).

Enfim, a aplicação de atividades gamificadas possibilita o desenvolvimento do ensino e da aprendizagem, bem como permite a melhoria da interação entre professores e alunos no ambiente de sala de aula, visto que os conteúdos são passados de forma interativa e dinâmica e não necessariamente utilizando tecnologias, pois pode-se fazer uso de elementos de jogos sem precisar de ambientes virtuais de aprendizagem.

\section{METODOLOGIA}

Esta pesquisa se caracteriza como exploratória quantitativa, com a utilização de bibliometria. Para tanto, foi realizada uma análise bibliométrica de artigos publicados com acesso livre, relacionados à utilização da gamificação, sendo utilizadas as bases de dados on-line da Scopus e Scielo para realizar a busca por produções científicas sobre o tema.

Na base Scopus, foi utilizada para busca a palavra-chave gamification no campo Article Title, Abstract, Keywords. Foi aplicado um filtro selecionando-se apenas artigos de acesso livre entre $2012 \mathrm{a}$ 2019, sendo encontradas 564 publicações sobre o tema.

Por sua vez, na base Scielo, foi utilizada a palavra-chave gamificação em pesquisa avançada, sendo encontrados apenas 15 artigos entre 2014 a 2019. A Figura 2 apresenta o processo de análise dos artigos encontrados. 
Figura 1 -Processo de análise dos artigos

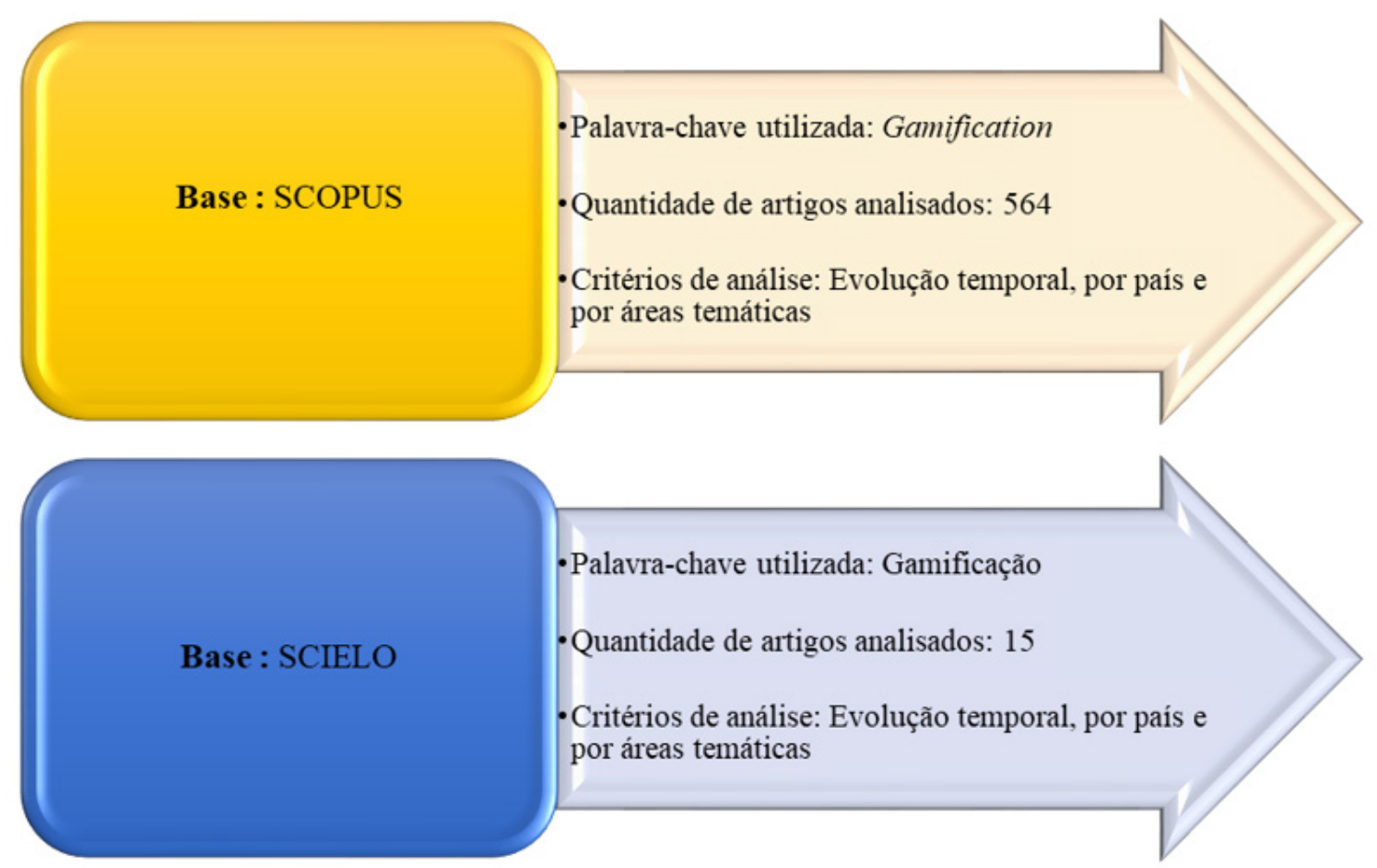

Fonte: Autores (2020)

Por sua vez, após o levantamento das informações encontradas na busca realizada na base Scopus e Scielo, os dados foram analisados priorizando-se a evolução temporal, os autores e os países que mais possuíam publicações sobre o tema em estudo. Além disso, foi utilizado o programa VOSviewer, permitindo-se a criação de mapas bibliométricos por meio da análise dos artigos encontrados.

\section{RESULTADOS E DISCUSSÕES}

\section{$6.1-$ Scielo}

A pesquisa realizada na base Scielo destaca que foram encontrados apenas 15 artigos relacionados à gamificação, sendo estes entre 2014 a 2019. No entanto, nota-se que não foram encontrados documentos anteriores ao ano de 2014 sobre o tema estudado, conforme apresentado na Tabela 1.

Tabela 1 - Distribuição de artigos por ano (2014-2019)

\begin{tabular}{cc}
\hline Ano de Publicação & Quantidade \\
\hline 2014 & 1 \\
\hline 2017 & 6 \\
\hline 2018 & 4 \\
\hline 2019 & 4 \\
\hline Total & $\mathbf{1 5}$ \\
\hline
\end{tabular}

Fonte: Autores. Baseado em dados da base Scielo (2020) 
É importante ressaltar que, embora as publicações sobre gamificação sejam recentes, a interação com os games no Brasil tiveram início com a chegada do Atari 2600, na década de 1980 (ALVES, MINHO, DINIZ, 2014), o que evidencia que os games já eram utilizados, porém não havia a aplicação de elementos de jogos nas atividades educacionais.

A Tabela 2 evidencia que Portugal e Brasil apresentaram maior número de artigos sobre o tema, com 5 cada um. É importante ressaltar que esse baixo número de países apresentados pode estar relacionado ao fato de a busca ter ocorrido apenas na base Scielo.

Tabela 2 - Publicações por país

\begin{tabular}{cc}
\hline Ano de Publicação & Quantidade \\
\hline Portugal & 5 \\
\hline Brasil & 5 \\
\hline Colômbia & 3 \\
\hline Costa Rica & 1 \\
\hline Peru & 1 \\
\hline Total & $\mathbf{1 5}$ \\
\hline
\end{tabular}

Fonte: Autores. Baseado em dados da base Scielo (2020)

A Tabela 3 apresenta as publicações por área. Foram destacadas 5 áreas, sendo que Ciências Sociais Aplicadas se sobressaiu às demais, evidenciando que os artigos, em sua maioria, estão relacionados a essa área.

Tabela 3 - Publicações por áreas temáticas

\begin{tabular}{cc}
\hline Ano de Publicação & Quantidade \\
\hline Ciências Sociais Aplicadas & 6 \\
\hline Ciências Humanas & 4 \\
\hline Linguística, Letras e Artes & 3 \\
\hline Engenharias & 2 \\
\hline Ciências da Saúde & 2 \\
\hline Total & 17 \\
\hline
\end{tabular}

Fonte: Autores. Baseado em dados da base Scielo (2020)

A seguir, destaca-se a análise realizada com os artigos relacionados à gamificação encontrados na base Scopus, evidenciando que essa base apresentou um número maior de artigos, comparada à base Scielo.

\section{2 - Scopus}

Na pesquisa realizada na base Scopus, foram analisados 564 artigos entre 2012 a 2019, evidenciando um crescimento na produção científica relacionada à gamificação nos últimos anos, visto o aumento do número de publicações destacados na Figura 2. 
Figura 2 - Distribuição de artigos por ano (2012 - 2019)

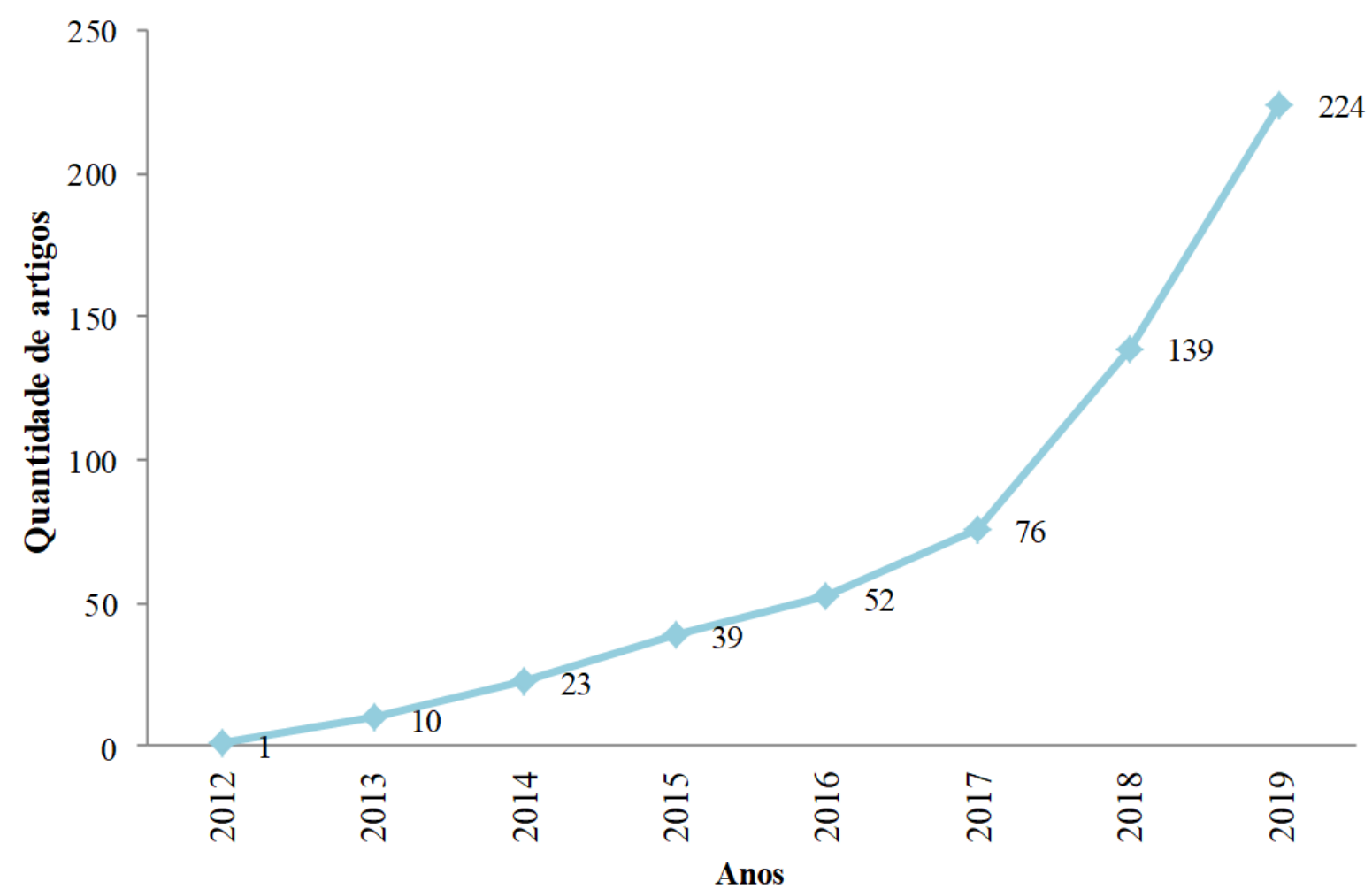

Fonte: Autores. Baseado em dados da base Scopus (2020)

Identifica-se, na Figura 2, que o ano de 2019 apresentou o maior número de publicações sobre o tema (224), representando cerca de 39,78\% dos resultados encontrados. Analisando o intervalo de 2015 a 2019, observa-se que foram publicados 530 artigos, totalizando $94,13 \%$ das publicações, o que mostra que houve um aumento significativo de publicações referentes ao tema nos últimos anos. 
Figura 3 - Publicações por áreas temáticas

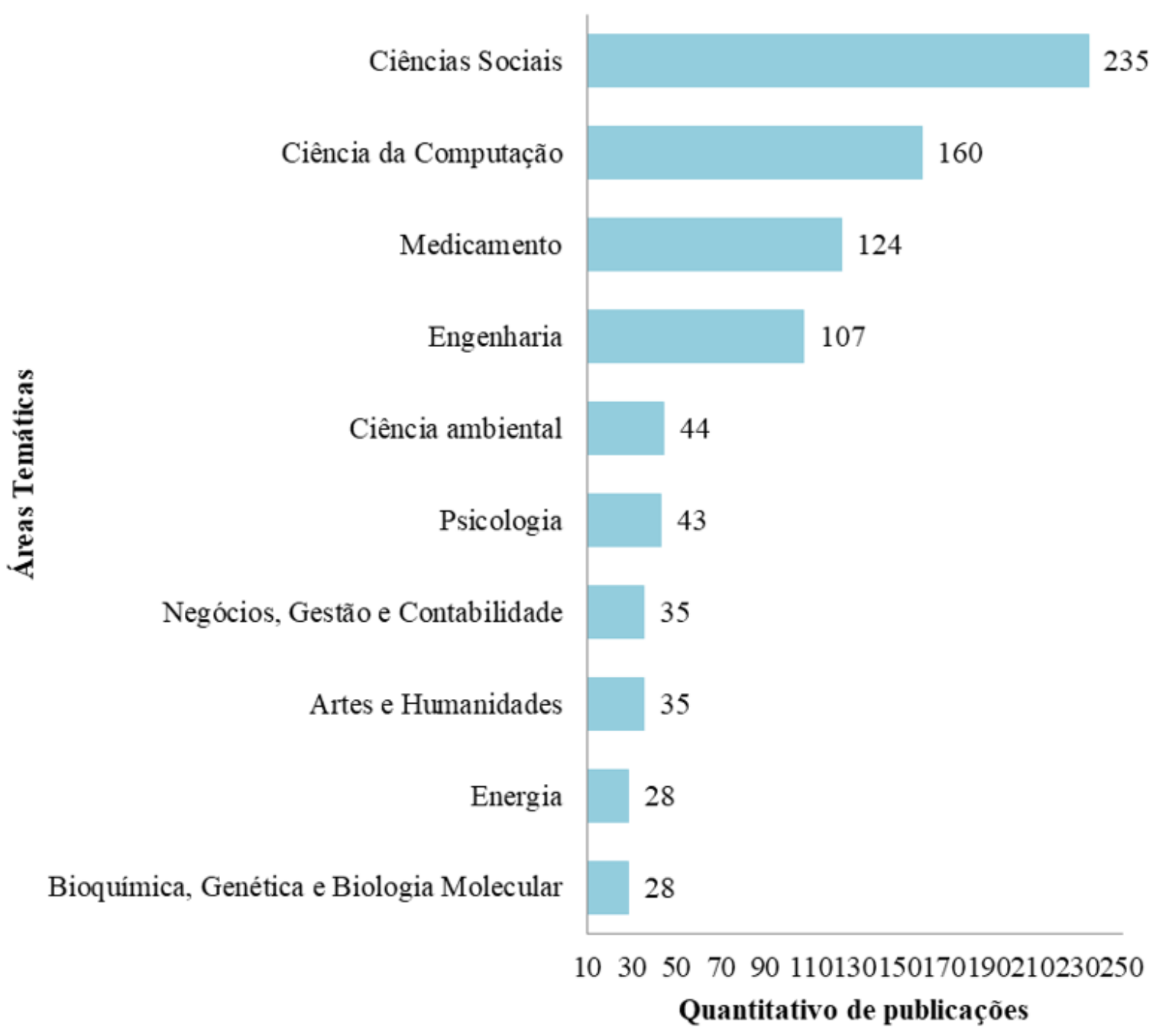

Fonte: Autores. Baseado em dados da base Scopus (2020)

Analisando os dados obtidos na Scopus, identificou-se as áreas temáticas com mais publicações sobre o tema. Destaca-se, com 235 (28\%), a área de Ciências Sociais; seguida da Ciência da Computação, com 160 (19,07\%); Medicamentos, com 124 (14,77\%); e Engenharia, com 107 (12,75\%). Observou-se que a área de Negócios, Gestão e Contabilidade está entre as 10 áreas com mais produções voltadas ao tema gamificação como ferramenta para melhoria do ensino, apresentando 35 publicações $(41,71 \%)$. 
Figura 4 - Países com mais publicações

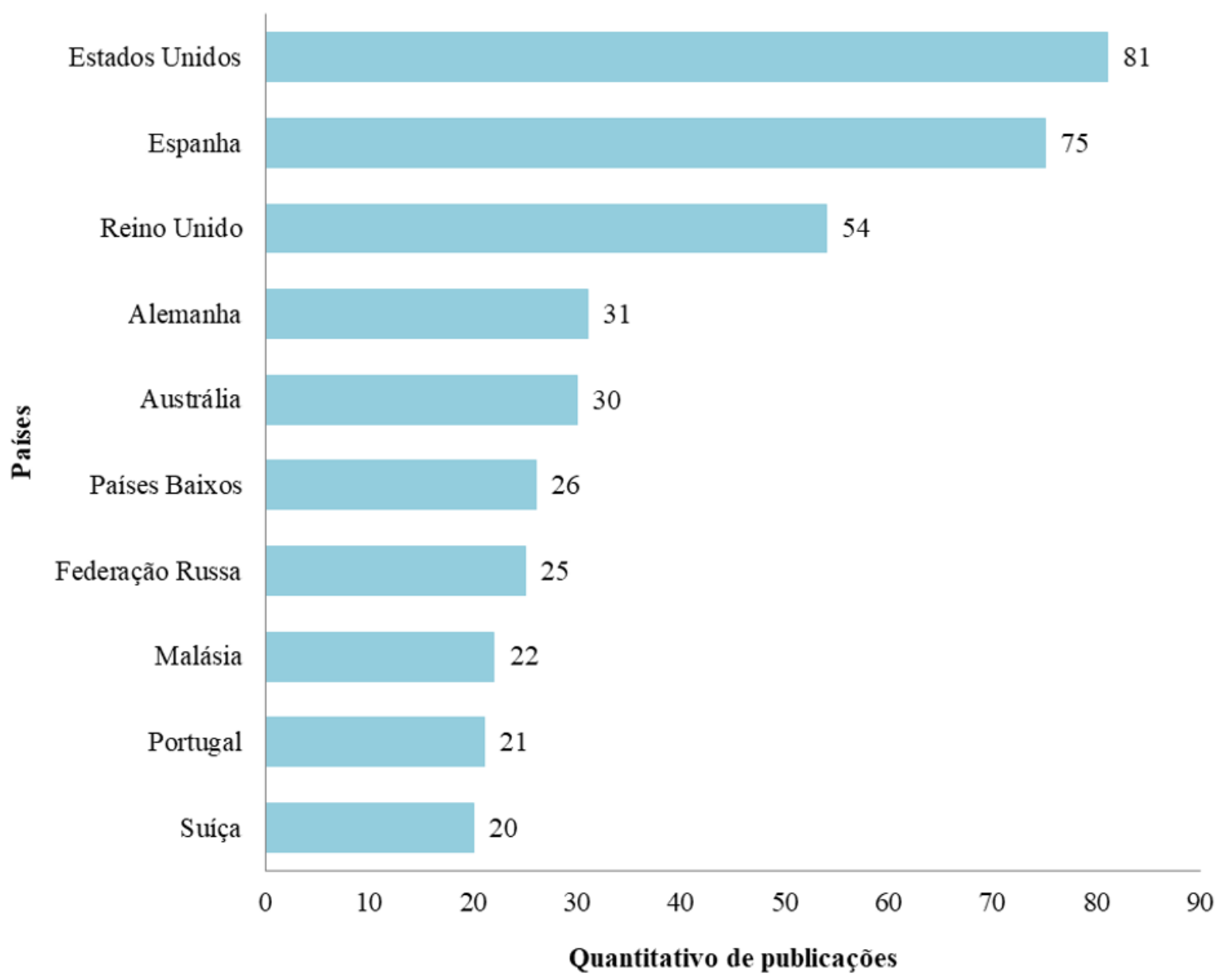

Fonte: Autores. Baseado em dados da base Scopus (2020)

A Figura 4 destaca que há uma concentração de publicações dos Estados Unidos (81), que representa 21,03\%; seguido da Espanha, com 75 (19,48\%); e do Reino Unido com 54 (14,02\%). Os países que apresentaram menor número de produção entre os 10 países com maior número de publicações sobre a gamificação foram: Portugal, com 21 publicações (5,45\%); e Suíça, com 20 publicações (5,19\%).

No entanto, o Brasil, está presente na relação de países que publicaram sobre o tema, porém não entre os dez países que realizaram mais publicações voltadas à gamificação, apresentando apenas 5 publicações.

Todavia, é importante ressaltar que o Brasil vem utilizando a gamificação no ensino das línguas, conforme abordam Leffa e Pinto (2014, p. 360), quando registram que "estudos realizados no ensino de línguas têm mostrado que os games podem contribuir significativamente para sua aprendizagem, principalmente nos estágios iniciais". 
Figura 5 - Ocorrências de palavras-chave

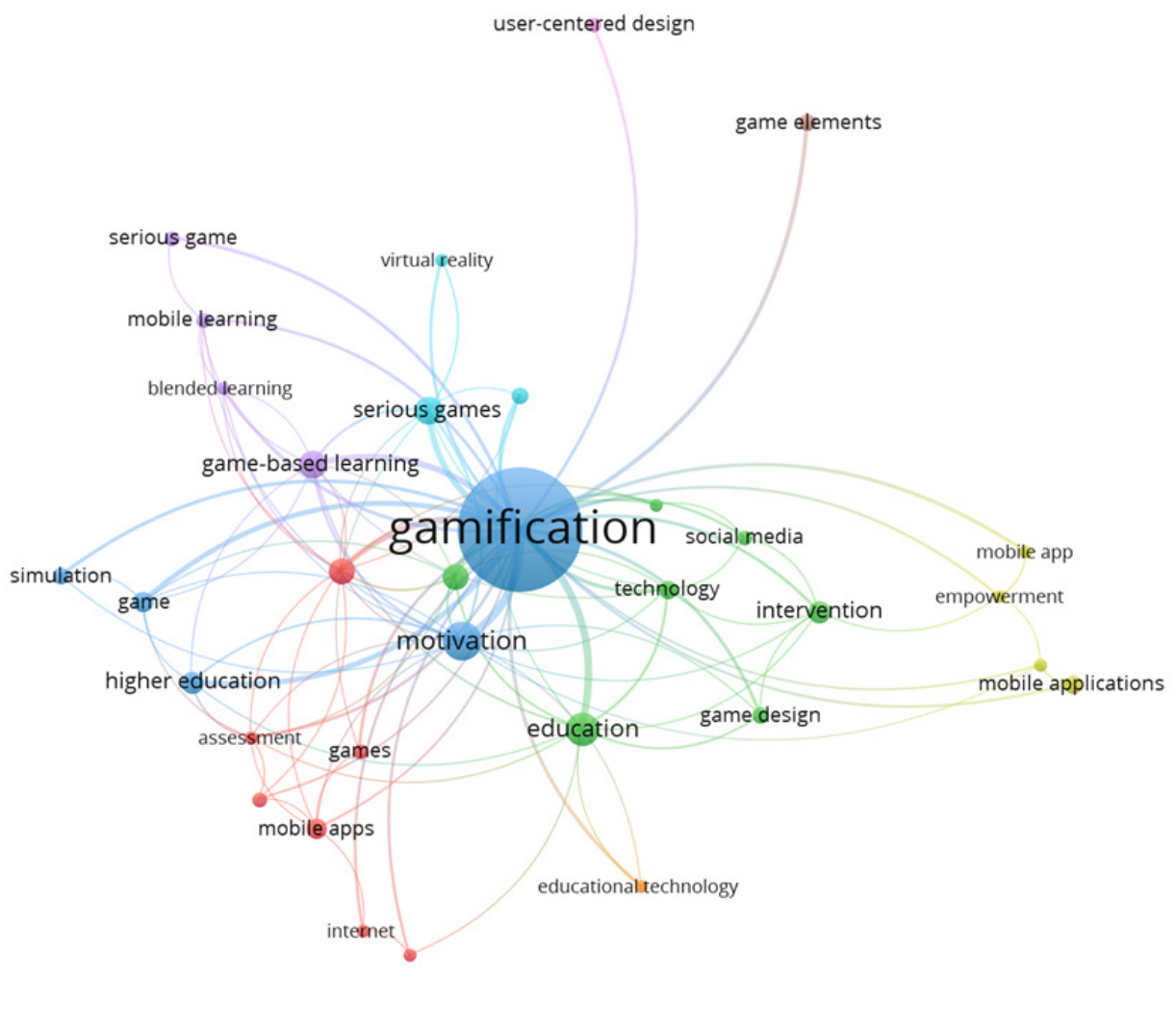

Fonte: VOSViewer (2020)

A Figura 5 evidencia a utilização do VOSviewer, este corresponde a um programa de computador que possui acesso livre e possibilita a criação de mapas bibliométricos. No caso desta pesquisa, buscou-se construir uma rede de ocorrências de palavras-chave que foram utilizadas nos 516 encontrados na base Scopus.

Em relação ao VOSviewer, identificou-se a frequência das palavras-chaves, sendo encontradas 54 . Desse número, foram retiradas todas as palavras que não eram relacionadas a proposta deste estudo, restando 33 palavras que formaram 5 clusters, que estão representados por diferentes cores, retratando a divisão de aglomerados entre as palavras. 0 mapa bibliométrico criado enfatiza palavras relacionadas à gamificação, mostrando que o levantamento realizado identificou estudos que abordam essa técnica.

A Figura 5 evidencia a utilização do VOSviewer, programa de computador que possui acesso livre e possibilita a criação de mapas bibliométricos. No caso desta pesquisa, buscou-se construir uma rede de ocorrências de palavras-chave que foram utilizadas nos 516 encontrados na base Scopus.

Em relação ao VOSviewer, identificou-se a frequência das palavras-chave, sendo encontradas 54 . Desse número, foram retiradas todas as palavras que não eram relacionadas à proposta deste estudo, restando 33 palavras que formaram 5 clusters, que estão representados por diferentes cores, retratando a divisão de aglomerados entre as palavras. 0 mapa bibliométrico criado enfatiza palavras relacionadas à gamificação, mostrando que o levantamento realizado identificou estudos que abordam essa técnica. 


\section{CONSIDERAÇÕES FINAIS}

A pesquisa utilizou a bibliometria para quantificar as publicações relacionadas à gamificação. Esse tipo de metodologia possibilita identificar periódicos e artigos sobre determinado tema, o que favorece o desenvolvimento de trabalhos futuros, tais como artigos científicos.

Foram encontradas poucas publicações na base Scielo, diferentemente da base Scopus, na qual foram identificadas diversas publicações de artigos científicos em relação à utilização da gamificação.

Além disso, a pesquisa possibilitou identificar que houve uma evolução no número de publicações sobre o tema, sendo que, em 2019, evidenciou-se um maior número de publicações sobre a gamificação. No entanto, notou-se que o Brasil apresentou poucos artigos sobre o tema, enquanto os Estados Unidos, Espanha e Reino Unido superaram o país com publicações sobre o tema.

Ademais, utilizou-se o programa VOSviewer para se criar um mapa bibliométrico com palavras-chave relacionadas ao levantamento dos artigos encontrados na Scopus, mostrando que a pesquisa buscou estudos relacionados à gamificação e sua utilização no ambiente escolar.

Como apresentado neste artigo, a gamificação utilizada na educação tende a incentivar o processo de compartilhamento presente na aprendizagem de grupos, uma vez que esse tipo de atividade faz com que o aluno se sinta mais estimulado a inovar, interagir e dividir conhecimentos com os demais colegas.

Apesar dos conceitos apresentados no artigo serem muito novos no contexto educacional, a gamificação surge para agregar mais valor à execução das tarefas exercidas pelos estudantes nas atividades propostas pelos professores, facilitando o processo de ensino e e de aprendizagem.

Ao analisar os artigos levantados, sugere-se a aplicação de atividades gamificadas para auxiliar no ensino e aprendizagem dos alunos, utilizando-se elementos de jogos nas disciplinas, visando à ampliação dos conteúdos apresentados e à dinamização do conhecimento das disciplinas estudadas em sala de aula.

Dessa forma, a partir desta pesquisa, espera-se contribuir para ampliação do debate sobre a gamificação na melhoria do ensino e aprendizagem, bem como explorar o debate sobre o engajamento que a gamificação proporciona ao ensino. Além disso, os resultados encontrados no levantamento de informações possibilitam maior familiaridade aos professores e alunos em relação ao tema.

\section{REFERÊNCIAS}

ALVES, Flora. Gamification: como criar experiências de aprendizagens engajadoras. Um guia completo: do conceito à prática. 2. ed. São Paulo: DVS, 2015.

ALVES, L. R. G.; MINHO, M.; Diniz, Marcelo. Gamificação: diálogos com a educação. In: Luciane Maria Fadel, Vania Ribas Ulbricht, Claudia Regina Batista e Tarcísio Vanzin.. (Org.). Gamificação na educação. 1. ed. São Paulo: Pimenta Cultural, 2014, v. 1, p. 73-95.

DETERDING, S.; DIXON, D.; KHALED, R.; NACKE, L. From game design elements to gamefulness: defining gamification. In: Proceedings of the 15th International Academic Mindtreck Conference, 2011.

FARDO, M.L. A gamificação aplicada em ambientes de aprendizagem. Novas Tecnologias da Educação, v. 11, n. 1, p. 1-9. 2013. 
FORMANSKI, F. N.; ALVES, J. B. Gamificação Aplicada à Aprendizagem de Grupo. In: XI Congresso Nacional de Excelência em Gestão, 2015.

INHELORA K. J. Uma Introdução ao Pensamento de Vygotsky. Revista Linhas, v. 3, n. 1, 2002.

GROH, F. Gamification: State of the art definition and utilization. Research Trends in Media Informatics. p. 39-46. 2012.

KAPP, Karl. The gamification of learning and instruction: game-based methods and strategies for training and education. San Francisco: Pfeiffer, 2012.

LEFFA, V. J.; PINTO, C. M. Aprendizagem como vício: o uso de games na sala de aula. Revista (Con) Textos Linguísticos, v.8, n. 10.1, p. 358-378, 2014.

ORLANDI, T. R. C.; DUQUE, C. G.; MORI, A. M.; ORLANDI, M. T. A. L. Gamificação: uma nova abordagem multimodal para a educação. Biblios, n. 70, p. 17-30, 2018.

PANTOJA, A. S.; PEREIRA, L. M. Gamificação: como jogos e tecnologias podem ajudar no ensino de idiomas. Estudo de caso: uma escola pública do Estado do Amapá. Estação Científica (UNIFAP), v. 8, n. 1, p. 111-120, 2018.

SALAMI, A. D. G.; SILVA, E. R.; TISOTT, P. B.; DIAS, D. T. A. D.; BOCCHESE, P. Gamificação: a Proposição de um Modelo para Aplicação nas Empresas. In: XVIII Mostra de Iniciação Científica, Pós-Graduação, Pesquisa e Extensão, Programa de Pós Graduação em Administraçaõ - UCS, 2018.

SHELDON, L. The Multiplayer Classroom: Designing Coursework as a Game. Boston, MA: Cengage Learning, 2012.

SILVA, M. R.; HAYASHI, C. R. M.; HAYASHI, M. C. P. I. Análise bibliométrica e cientométrica: desafios para especialistas que atuam no campo. InCID: Revista de Ciência da Informação e Documentação, v. 2, n. 1, p. 110-129, jan./jun. 2011.

TOLOMEI, B.V. A Gamificação como Estratégia de Engajamento e Motivação na Educação, EAD em Foco, v. 7, n. 2, p. 145-156, 2017.

WU, M. The Science of Gamification. 2011. Disponível em: http://fora.tv/2011/09/16/Michael_Wu_The_ Science_of_Gamification. Acesso em: 20 abr. 2020.

VIANNA, Y.; VIANNA, M.; MEDINA, B.; TANAJA, S. Gamification, Inc: como reinventar empresas a partir de jogos. 1. ed. Rio de Janeiro: MJV Press, 2013. 